\title{
DETERMINAÇÃO DE COMPOSTOS FENÓLICOS E AVALIAÇÃO DA ATIVIDADE ANTIOXIDANTE DE Lafoensia pacari (LYTHRACEAE)
}

DETERMINATION OF PHENOLIC AND EVALUATION OF ANTIOXIDANT ACTIVITY OF Lafoensia pacari (LYTHRACEAE)

DETERMINACIÓN DE LOS FENÓLICOS Y EVALUACIÓN DE LA ACTIVIDAD ANTIOXIDANTE DE Lafoensia pacari (LYTHRACEAE)

\author{
Renan Kubiachi Burque ${ }^{1 *}$, Lenise Petter Francesconi ${ }^{1}$, André Tavares \\ Victorino ${ }^{1}$, Marcello Ávila Mascarenhas ${ }^{1}$, Keila Maria Ceresér ${ }^{1}$ \\ ${ }^{1}$ Centro Universitário Metodista - IPA, Porto Alegre, RS. Endereço: Rua Coronel Joaquim \\ Pedro Salgado, 80, Curso de Farmácia, CEP 90420-060, Porto Alegre, RS. \\ E-mail*: renan_kade2@hotmail.com
}

Submetido em: 17/06/2013; Aceito em: 22/04/2015

RESUMO: A espécie Lafoensia pacari A. St.-Hil (Lythraceae) é uma planta do cerrado brasileiro, encontrada na Bahia, Goiás e Maranhão. Empregada popularmente para problemas gástricos e inflamação, conhecida por mangava brava, dedaleiro e/ou pacari. O objetivo do trabalho foi determinar a concentração de compostos fenólicos e avaliar a atividade antioxidante do extrato hidroalcoólico a $70 \%$ e frações.A planta foi coletada no município de Estreito-MA, das folhas secas foi obtido o extrato hidroalcoólico a $70 \%$ e separadas frações pelos solventes hexano, clorofórmio, acetato de etila e metanol.Dos extratos foram determinadas as concentrações de fenois (reagente Folin-Ciocalteau), flavonoides (reação com cloreto de alumínio), ácidos fenólicos (diferença entre as quantidades dosadas de fenois e flavonoides) e avaliado a atividade antioxidante pelo método in vitro 2,2- 


\section{- Revista Eletrônica de Farmácia}

FIRMO et al.

REF-ISSN1808-0804 Vol.XII (1),1-10, 2015.

difenil-1-picril-hidrazila (DPPH). Os resultados demonstraram que o extrato bruto, a fração acetato de etila e metanólica apresentaram altos teores de compostos fenólicos e atividade antioxidante para o extrato metanólico uma vez que o valor da concentração efetiva para se obter $50 \%$ da atividade $\left(C_{50}\right)$ foi de $19,69 \mu \mathrm{g} / \mathrm{mL}$. Estes resultados caracterizam a $L$. pacari como matéria-prima natural com ação antioxidante, podendo esta atividade estar relacionada à presença de constituintes fenólicos, substâncias com este potencial.

PALAVRAS-CHAVE: antioxidantes, flavonoides, Lafoensia pacari, Lythraceae.

ABSTRACT: The species Lafoensia pacari A. St.-Hil (Lythraceae) is a Brazilian cerrado plant, found in Bahia, Goiás and Maranhão. Popularly used for stomach problems and inflammation, known as mad mangava, dedaleiro and/or pacari. The objective was to determine the concentration of phenolic compounds and the antioxidant activity of the hydroalcoholic extract $70 \%$ and fractions. The plant was collected in the municipality of Estreito-MA, the dried leaves was obtained alcoholic extract to $70 \%$ and separated fractions by solvent hexane, chloroform, ethyl acetate and methanol. The extracts were determined concentrations of phenols (Folin-Ciocalteu reagent), flavonoids (reaction with aluminum chloride), phenolic acids (difference between the amounts of phenols and flavonoids dosed) and evaluated the antioxidant activity by the in vitro 2,2 diphenyl-1-picryl-hidrazila (DPPH). The results demonstrated that the crude extract, ethyl acetate fraction and methanol showed high levels of phenolics and antioxidant activity for methanol extract since the value of the effective concentration to achieve $50 \%$ of the activity $\left(E_{50}\right)$ was $19,69 \mu \mathrm{g} / \mathrm{mL}$. These results characterize L. pacari as natural raw material with antioxidant activity, which may be related activity to the presence of phenolic constituents, substances with this potential.

KEYWORDS: antioxidants, flavonoids, Lafoensia pacari, Lythraceae.

RESUMEN: Las especies Lafoensia pacari A. St.-Hil (Lythraceae) es una planta cerrado brasileño, que se encuentra en Bahía, Goiás y Maranhão. Popularmente se utiliza para problemas de estómago y la inflamación, conocidos como mangava loco, dedaleiro y/o Pacari. El objetivo fue determinar la concentración de compuestos fenólicos y la actividad antioxidante del extracto hidroalcohólico $70 \%$ y fracciones. La planta se recogió en el municipio de Estreito-MA, las hojas secas se 


\section{- Revista Eletrônica de Farmácia}

FIRMO et al.

REF-ISSN1808-0804 Vol.XII (1),1-10, 2015.

obtuvo el extracto alcohólico de $70 \%$ y se separan las fracciones por solvente hexano, cloroformo, acetato de etilo y metanol. Los extractos se determinaron las concentraciones de fenoles (reactivo de Folin-Ciocalteu), flavonoides (de reacción con cloruro de aluminio), ácidos fenólicos (diferencia entre las cantidades de fenoles y flavonoides dosificadas) y se evaluaron la actividad antioxidante in vitro por el 2,2 difenil-1-picrilo-hidrazila (DPPH). Los resultados demostraron que el extracto crudo, la fracción de acetato de etilo y metanol mostraron altos niveles de compuestos fenólicos y actividad antioxidante de extracto de metanol ya que el valor de la concentración eficaz para alcanzar el $50 \%$ de la actividad $\left(\mathrm{EC}_{50}\right)$ era $19,69 \mu \mathrm{g} / \mathrm{mL}$. Estos resultados caracterizan L. Pacari como materia prima natural con actividad antioxidante, que puede estar relacionado con la actividad a la presencia de constituyentes fenólicos, sustancias con este potencial.

PALABRAS CLAVE: antioxidantes, flavonoides, Lafoensia pacari, Lythraceae.

\section{INTRODUÇÃO}

O conhecimento sobre plantas medicinais no tratamento e na cura de enfermidades é tão antigo quanto à espécie humana, simboliza muitas vezes o único recurso terapêutico de muitas comunidades e grupos étnicos ${ }^{(1)}$.

Dentre essas, a espécie vegetal Lafoensia pacari A. St.-Hil. (Lythraceae) é uma planta arbórea, com 5 a $15 \mathrm{~m}$ de altura e 20 a $40 \mathrm{~cm}$ de diâmetro, encontrada na América Central e América do Sul, também é observada no cerrado brasileiro ${ }^{(2)}$, nos estados da Bahia, Goiás, Minas Gerias, Maranhão, Mato Grosso e Distrito Federal ${ }^{(3)}$. Conhecida popularmente por "mangaba brava", "pacari" ou "dedaleiro" é usada na

medicina tradicional brasileira ${ }^{(4)}$,
pelos métodos de infusão e/ou
maceração utilizando as folhas e a
casca, e administrada por via oral
para o tratamento de câncer,
distúrbios gástricos, inflamação e
cicatrização ${ }^{(5)}$.
Sobre essa planta, estudos
fitoquímicos evidenciaram que os
principais compostos ativos
encontrados na planta são os taninos,
flavonoides, saponinas, esteroides,
triterpenoides e alcaloides ${ }^{(6)}$; já em
outros trabalhos, avaliando a
atividade de L. pacari, foi possível
observar efeito antimicrobiano(7), anti-
inflamatório, analgésico ${ }^{(8)}$,
antiulcerogênico ${ }^{(9)}$, antioxidante ${ }^{(5)}$,
antidepressivo ${ }^{(4)}$, demonstrando o
potencial farmacológico desta planta.


FIRMO et al.

REF-ISSN1808-0804 Vol.XII (1),1-10, 2015.

\begin{abstract}
Destaca-se que o uso de antioxidantes naturais tem aumentado com as descobertas das propriedades dos componentes que são produzidos pelas plantas através do metabolismo secundário. Atribui-se à presença de compostos fenólicos, com destaque aos flavonoides, a atividade antioxidante dos componentes produzidos pelos vegetais. Esses componentes podem atuar como agentes redutores, sequestradores de radicais livres, quelantes de metais ou desativadores do oxigênio singleto e/ou exibir, simultaneamente, mais de uma dessas funções ${ }^{(10)}$.

Diante deste contexto, o presente trabalho tem como objetivo determinar os compostos fenólicos e avaliar a atividade antioxidante do extrato bruto e frações da planta $L$. pacari.
\end{abstract}

\section{MATERIAIS E MÉTODOS}

\section{Coleta do material vegetal}

As amostras de $L$. pacari foram coletadas no Parque Nacional da Chapada das Mesas, no município de Estreito-MA, em julho de 2012; sua exsicata foi preparada e depositada no Herbário Ático Seabra da Universidade Federal do MaranhãoUFMA sob o número 1490.

\section{Preparo do extrato e frações do material vegetal}

As folhas frescas (pelos menos $500 \mathrm{~g}$ ) foram levadas à estufa a $35^{\circ} \mathrm{C}$ durante 24 horas. Após secagem as amostras foram trituradas em moinho, obtendo um pó moderadamente fino, que foi submetido à extração com álcool a $70 \%$ durante 7 dias. O extrato foi concentrado sobre pressão reduzida em um evaporador rotativo, armazenado em frasco com rótulo e tampa de rosca. Após essa etapa, o extrato bruto, assim obtido, foi submetido a fracionamento com extração sequencial com hexano, clorofórmio, acetato de etila e metanol. De cada fração adquirida, o solvente foi evaporado até à secura e realizados os ensaios.

\section{Determinação de flavonoides, fenois e ácidos fenólicos}

A determinação das concentrações de polifenois totais foi obtida utilizando reagente de FolinCiocalteau (Merck) e carbonato de sódio a $20 \%$, sendo medido através de análise espectrofotométrica em espectrofotômetro UV-Vis (Lambda 35, Perkin Elmer) a 760nm. 
FIRMO et al.

REF-ISSN1808-0804 Vol.XII (1),1-10, 2015.

Concentrações de ácido gálico (Merck) foram utilizadas como padrões e os resultados expressos em porcentagem de ácido gálico. Para concentração de flavonoides totais, foi aplicado 0 método colorimétrico, utilizando solução metanólica de cloreto de alumínio $\left(\mathrm{AlCl}_{3}\right)$ a $5 \%$, realizando a leitura das amostras em espectrofotômetro UV-Vis (Lambda 35, Perkin Elmer) a 425nm. Concentrações de quercetina (Merck) foram utilizadas como padrões, e os resultados expressos em porcentagem de quercetina ${ }^{(11)}$. A concentração de ácidos fenólicos totais foi determinada pela diferença entre as quantidades dosadas de polifenois e flavonoides totais $^{(12)}$. Os teores de polifenois e flavonoides totais dos extratos foram realizados em triplicata e expressos como média \pm desvio padrão.

\section{Determinação da atividade antioxidante pelo método de DPPH \\ Para avaliação da atividade} antioxidante, foi utilizado o método fotocolorimétrico in vitro, utilizando o radical livre estável 2,2-difenil-1picrilhidrazila (DPPH) (Sigma-Aldrich) com solução metanólica de DPPH $(40 \mu \mathrm{g} / \mathrm{mL})$ em metanol P.A. (Merck) $^{(13)}$. A partir das soluções dos extratos de L. pacari, na concentração de $100 \mu \mathrm{g} / \mathrm{mL}$ (solução estoque), foram obtidas soluções metanólicas em diferentes concentrações $(5,10$, 25 e $50 \mu \mathrm{g} / \mathrm{mL}$ ), de onde se retirou uma alíquota de $0,5 \mathrm{~mL}$ e adicionou-se $3,5 \mathrm{~mL}$ da solução de DPPH. Como controle negativo, foi utilizado o metanol P.A. Trinta minutos após a adição de DPPH, foi realizada a leitura em espectrofotômetro UV-Vis (Lambda 35, PerkinElmer) a 517nm. As análises foram realizadas em triplicata e a percentagem da atividade sequestradora (\%AS) foi calculada pela equação:

$$
\begin{gathered}
\% \mathrm{AS}=100 \times\left(A_{\text {controle }}-A_{\text {amostra }}\right) / \\
A_{\text {controle }}
\end{gathered}
$$

Onde $A_{\text {controle }}$ é a absorvância do controle (solução com radical DPPH e metanol) e $A_{\text {amostra é a absorvância }}$ do radical na presença dos extratos.

\section{RESULTADOS E DISCUSSÃO}

Antioxidantes são agentes que retardam ou previnem as lesões causadas pelos radicais livres nas células $^{(14)}$, é qualquer substância que, presente em baixas concentrações quando comparada ao substrato oxidável, atrasa ou inibe a oxidação deste substrato de maneira eficaz ${ }^{(10)}$.

Os radicais formados a partir de antioxidantes não são reativos para propagar a reação em cadeia que 


\section{- Revista Eletrônica de Farmácia}

FIRMO et al.

REF-ISSN1808-0804 Vol.XII (1),1-10, 2015.

seria prejudicial à célula, eles são neutralizados por reação com outro radical, formando produtos estáveis ou podem ser reciclados por outro antioxidante ${ }^{(15)}$.

Dentre as diversas classes de substâncias antioxidantes de ocorrência natural, os compostos fenólicos têm recebido muita atenção nos últimos anos, sobre tudo por inibirem a peroxidação lipídica e a lipooxigenase in vitro(16).

Os compostos fenólicos de plantas enquadram-se em diversas categorias como fenois simples, ácidos fenólicos (derivados de ácidos benzoicos e cinâmico), cumarinas, flavonoides, estilbenos, taninos condensados e hidrolisáveis, lignanas e ligninas ${ }^{(17)}$.

Na Tabela 1, está apresentada a quantificação dos flavonoides, fenois e ácidos fenólicos do extrato bruto e frações da planta $L$. pacari.

Tabela 1.Teores de flavonoides, fenois e ácidos fenólicos totais do extrato bruto e frações de L. pacari obtidos por maceração.

\begin{tabular}{lccc}
\hline & FLAVONOIDES (\%) $^{\mathbf{a}, \mathbf{b}}$ & FENOIS (\%) $^{\mathbf{a}, \mathbf{c}}$ & $\begin{array}{c}\text { ÁCIDOS } \\
\text { FENóLICOS (\%) }\end{array}$ \\
\hline EHB & $15,7 \% \pm 0,02$ & $29,6 \% \pm 0,5$ & $13,9 \% \pm 0,5$ \\
\hline EFH & $6,6 \% \pm 0,3$ & $8,3 \% \pm 0,2$ & $1,6 \% \pm 0,1$ \\
\hline EFC & $11,3 \% \pm 0,3$ & $13,7 \% \pm 0,3$ & $2,3 \% \pm 0,8$ \\
\hline EFAE & $14,9 \% \pm 0,3$ & $28,5 \% \pm 0,3$ & $13,6 \% \pm 0,1$ \\
\hline EFM & $14,4 \% \pm 0,04$ & $28,6 \% \pm 0,5$ & $14,2 \% \pm 0,6$ \\
\hline
\end{tabular}

EHB = Extrato hidroalcoólico bruto de L. pacari; EFH = Extrato fração hexânica; EFC = Extrato fração clorofórmica; EFAE = Extrato fração acetato de etila; EFM = Extrato fração metanólica obtido por maceração. ${ }^{a}$ Resultados representam médias \pm desvio padrão $(n=3)$, ${ }^{\mathrm{b}}$ Expressos como equivalente de quercetina. ${ }^{c}$ Expressos como equivalente de ácido gálico.

Nota-se que, na Tabela 1 , os EHB, EFAE e EFM demonstraram maiores teores de compostos flavonoides, fenóis e ácidos fenólicos. Isso se deve pela afinidade destas substâncias por solventes polares, melhorando assim sua extração, pois a maioria dos compostos fenólicos não é encontrada no estado livre na natureza, mas sob forma de ésteres ou de heterosídeos; sendo, portando, solúveis em água e em solventes orgânicos polares ${ }^{(18)}$.

Alguns estudos fitoquímicos evidenciaram a presença de compostos ativos na espécie $L$. pacari 
FIRMO et al.

REF-ISSN1808-0804 Vol.XII (1),1-10, 2015.

entre eles, taninos, flavonoides, saponinas, esteroides, triterpenoides e alcaloides $^{(6)}$. Dentre estes, vários são compostos fenólicos o que corroboram com a presente pesquisa na determinação destas substâncias, os mesmos apresentam um alto potencial bioativo como antioxidante.
Considerando que substâncias naturais podem ser responsáveis pelo efeito de proteção contra os riscos de muitos processos patológicos, os resultados descritos neste trabalho estimulam a continuidade dos estudos para avaliar a ação antioxidante de $L$. pacari.

Figura 1.Relação da porcentagem de inibição do DPPH de acordo com a concentração do EBH e frações de L. pacari.

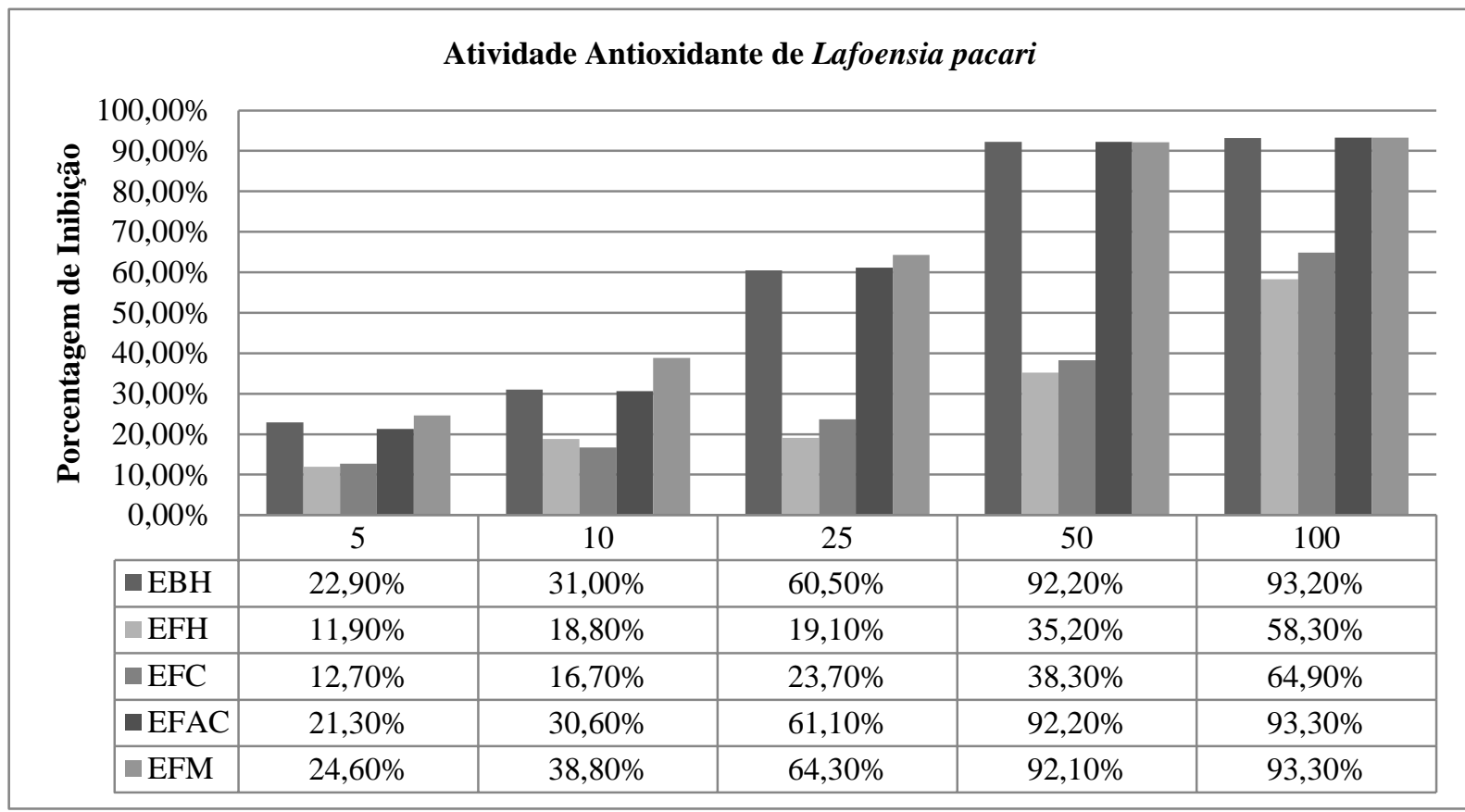

É possível observar na Figura 1, que os EBH, EFAE e EFM, foram os que inibiram em maior porcentagem o $\mathrm{DPPH}$; pois, quanto maior o consumo de DPPH pela amostra, maior é sua atividade antioxidante; sendo assim, quanto maior a concentração da amostra e menor a absorvância, maior o consumo de $\mathrm{DPPH}^{(19)}$.

A porcentagem de atividade antioxidante (\%AA) corresponde à quantidade de DPPH consumida pelo antioxidante, sendo que sua quantidade necessária para decrescer a concentração inicial de DPPH em 


\section{- Revista Eletrônica}

FIRMO et al.

REF-ISSN1808-0804 Vol.XII (1),1-10, 2015.

$50 \%$ é denominada concentração menor será a sua $\mathrm{CE}_{50}$ e maior a sua eficiente $\left(\mathrm{CE}_{50}\right)$. Quanto maior o atividade antioxidante ${ }^{(20)}$. consumo de DPPH por uma amostra,

Portanto nota-se, na Tabela 2, que o EFM foi o que apresentou um valor menor de $\mathrm{CE}_{50}$ para atividade antioxidante.

Tabela 2. Atividade antioxidante expressa em valor de $\mathrm{CE}_{50}$ do extrato bruto e frações obtido de L. pacari por maceração.

\begin{tabular}{lc}
\hline Maceração & $\mathbf{C E}_{\mathbf{5 0}}(\boldsymbol{\mu g} \mathbf{m} \mathbf{m L})$ \\
\hline EBH & 24,63 \\
\hline EFH & 82,97 \\
\hline EFC & 72,50 \\
\hline EFAE & 25,16 \\
\hline EFM & 19,69 \\
\hline
\end{tabular}

Isso indica que os compostos com atividade antioxidante concentram-se preferencialmente nos extratos mais polares (etanol e água), enquanto que os compostos mais apolares não apresentam atividade antioxidante significativa ${ }^{(21)}$.

Diante disso, percebe-se que o bom poder antioxidante dessa planta pode ser explicado pela presença de substâncias capazes de inibir os radicais livres. Estudos químicos de extratos alcoólicos de $L$. pacari resultaram no isolamento e identificação de ácido gálico e ácido elágico, compostos com potencial antioxidantes $^{(5)}$.

Em geral, a atividade é associada ao conteúdo fenólico presente nas plantas ${ }^{(22)}$. Sobre isso, o trabalho de Vieira et al. ${ }^{(23)}$ mostraram que a capacidade antioxidante de polpas de frutos tropicais está relacionado com a presença de teor de fenólicos nestas polpas, corroborando com a presente pesquisa e os achados na literatura.

\section{CONCLUSÃO}

Os resultados permitem considerar que a atividade antioxidante da planta $L$. pacari esta diretamente ligada aos seus constituintes químicos, principalmente, aos compostos fenólicos encontrados na mesma, pois os teores determinado destas substâncias foram consideravelmente 


\section{- Revista Eletrônica de Farmácia}

FIRMO et al.

REF-ISSN1808-0804 Vol.XII (1),1-10, 2015.

altos. Essa atividade concentra-se preferencialmente nos extratos polares (EBH, EFAC e EFM) das folhas, provavelmente, tendo como constituintes polifenois e flavonoides.

Portanto o conhecimento das propriedades antioxidantes da planta pode estimular e direcionar a continuidade da pesquisa em outros testes experimentais que estejam relacionados a esta ação

\section{AGRADECIMENTOS}

Agradecemos à Fundação de Amparo à Pesquisa e Desenvolvimento Científico do Maranhão (FAPEMA), à Coordenação de Aperfeiçoamento de Pessoal de Nível Superior (CAPES) e à Universidade Federal do Maranhão (UFMA).

farmacológica.

\section{REFERÊNCIAS}

1 - Silva RAO, Santos MRMC, Santos Filho FC, Silva MCC, Oliveira GAL, Silva MGO, Marques LGA. Prospecção tecnológica de fitoterápico (Euphorbia tirucalli L.) utilizado no tratamento de neoplasias e outras doenças. Cadernos de Prospecção., 2013;6(4):490-499.

2 - Cabral PRF, Pasa MC. Mangava-brava: Lafoensia pacari A. St. -Hil. (Lythraceae) e a etnobotânica em Cuiabá, MT. Revista Biodiversidade,2009;8(1):2-21.

3 - Carvalho PER. Espécies florestais brasileiras: recomendações silviculturais, potencialidade e uso da madeira. Brasilia: EMBRAPA, CNPF. Colombo, 1994.

4 - Galdino PM, Nascimento MVM, Sampaio BL, Ferreira RN, Paula JR, Costa EA. Antidepressant-like effect of Lafoensia pacari A. St.-Hil. ethanolic extract and fractions in mice. J Ethnopharmacol, 2009;124:581-585.

5 - Solon S, Lopes L, Sousa Júnior PT, Schmeda-Hirschmann G. Free radical scavenging activity of Lafoensia pacari. J Ethnopharmacol,2000;72:173-178.

6 - Violante IMP, Souza IM, Venturini CL, Ramalho AFS, Santos RAN, Ferrari M. Avaliação in vitro da atividade fotoprotetora de extratos vegetais do cerrado de Mato Grosso. Rev Bras Farmacogn, 2009;19(2A):452-57.

7 - Firmo WCA, Miranda MV, Coutinho GSL, Silveira LMS, Olea RSG. Estudo fitoquímico e avaliação da atividade antibacteriana de Lafoensia pacari (Lythraceae). Publ. UEPG Ci. Biol. Saúde., 2014;20(1):7-12.

8 - Rogerio AP, Fontanari C, Melo MC, Ambrosio SR, Souza GE, Pereira OS et al. Anti-inflammatory, analgesic and antioedematous effects of Lafoensia pacari extract and ellagic acid. J Pharm Pharmacol,2006;58:1265-1273. 


\section{1) Revista Eletrônica de Farmácia}

FIRMO et al.

REF-ISSN1808-0804 Vol.XII (1),1-10, 2015.

9 - Tamashiro Filho P. Avaliação da atividade antiúlcera do extrato bruto metanólico de Lafoensia pacari St. Hil. (Mangava brava) [dissertation]. Cuiabá: Universidade Federal de Mato Grosso; 1999.

10 - Halliwell B, Aeschbach R, Logiger J, Aruoma OI. The characterization of antioxidants. Food Chem. Toxicol. 1995;33(7): 601-617.

11 - Dutra RP, Nogueira AMC, Marques RRO, Costa MCP, Ribeiro MNS. Avaliação farmacognóstica de geoprópolis de Melipona fasciculata Smith (tiúba) em municípios da Baixada maranhense, Brasil.Rev Bras Farmacogn, 2008;18(4):557562.

12 - Woisky RGR, Salatino A. Analysis of propolis: some parameters and procedures for chemical quality control. J Apicult Res, 1998;37:99-105.

13 - Brand-Williams W, Cuvelier ME, Berset C. Use of a free radical method to evaluate antioxidant activity. Food Sci. Technol., 1995;28:25-30.

14 - Andrade ER, Melo-Sterza FA, Seneda MM, Alfieri AA. Consequências da produção das espécies reativas de oxigênio na reprodução e principais mecanismos antioxidantes. Rev. Bras. Reprod. Anim., 2010;34(2):79-85.

15 - Sousa CMM, Silva HR, Vieira Júnior GM, Ayres MCC, Costa LS, Araújo DS et al. Fenóis totais e atividade antioxidante de cinco plantas medicinais. Quím. Nova, 2007;30(2): 351-355.

16 - Soares S. E. Ácidos fenólicos como antioxidantes. Rev. Nutr., 2002;15(1):7181.

17 - Naczk M, Shahidi F. Extractions and analysis of phenolics in food. J. Chromatogr. A., 2004;1054(1-2):95- 111.

18 - Monteiro JM, Albuquerque UP, Araújo EL, Amorim ELC. Taninos: uma abordagem química à ecologia. Quím. Nova, 2005;28(5): 892-896.

19 - Nascimento JC, Lage LFO, Camargos CRD, Amaral JC, Costa LM, Sousa AN, Oliveira FQ. Determinação da atividade antioxidante pelo método DPPH e doseamento de flavonoides totais em extratos de folhas da Bauhinia variegata L. Rev. Bras. Farm., 2011;92(4):327-332.

20 - Borges LL, Lúcio TC, Gil ES, Barbosa EF. Uma abordagem sobre métodos analíticos para determinação da atividade antioxidante em produtos naturais. Enciclopédia Biosfera., 2011;7(12):1-20.

21 - Ribeiro SR, Fortes CC, Oliveira SCC, Castro CFS. Avaliação da atividade antioxidante de Solanum paniculatum (Solanaceae). Arq. Ciênc. Saúde Unipar, 2007;11(3):179-183.

22 - Silva MLC, Costa RS, Santana AS, Koblitz MGB. Compostos fenólicos, carotenoides e atividade antioxidantes em produtos vegetais. Semina: Ciências Agrárias., 2010;31(3):669-682. 
FIRMO et al.

REF-ISSN1808-0804 Vol.XII (1),1-10, 2015.

23 - Vieira LM, Sousa MSB, Mancini Filho J, Lima A. Fenólicos totais e capacidade antioxidante in vitro de polpas de frutos tropicais. Rev. Bras. Frutic., $2011 ; 33(3): 888-897$. 